\title{
Absorption Measurements of Cell Monolayers Relevant to Mechanisms of Laser Phototherapy: Reduction or Oxidation of Cytochrome c Oxidase Under Laser Radiation at $632.8 \mathrm{~nm}$
}

\author{
Tiina I. Karu, Dr. Sci., Ph.D., ${ }^{1}$ Ludmila V. Pyatibrat, M.S., ${ }^{1}$ \\ Sergei F. Kolyakov, M.S., ${ }^{2}$ and Natalya I. Afanasyeva, Ph.D. ${ }^{3}$
}

\begin{abstract}
Objective: The objective of this work was a further investigation of redox mechanisms of laser phototherapy on the cellular level. Background Data: Cytochrome c oxidase, the terminal enzyme of the mitochondrial respiratory chain, is believed to work as the photoacceptor to modulate cellular metabolism in laser phototherapy. Materials and Methods: The changes in the absorption spectra of HeLa-cell monolayers before and after irradiation at $632.8 \mathrm{~nm}$ using fast multi-channel recording were evaluated by the intensity ratio between the peaks at 770 and $670 \mathrm{~nm}$ (intensity ratio criterion). Results: By the intensity ratio criterion, the irradiation effects (reduction or oxidation of the photoacceptor) depended on the initial redox status of cytochrome c oxidase. The irradiation (three times at $632.8 \mathrm{~nm}$, dose $=6.3 \times 103 \mathrm{~J} / \mathrm{m}^{2}, \tau_{\text {irrad. }}=10 \mathrm{sec}, \tau_{\text {record. }}=600 \mathrm{msec}$ ) of cells initially characterized by relatively oxidized cytochrome c oxidase caused first a reduction of the photoacceptor, and then its oxidation (a bell-shaped curve). The irradiation by the same scheme of the cells with initially relatively reduced cytochrome c oxidase caused first oxidation and then a slight reduction of the enzyme (a curve opposite to the bell-shaped curve). Conclusion: The experimental results of our work demonstrate that irradiation at $632.8 \mathrm{~nm}$ causes either a (transient) relative reduction of the photoacceptor, putatively cytochrome c oxidase, or its (transient) relative oxidation, depending on the initial redox status of the photoacceptor. The maximum in the bell-shaped dose-dependence curve or the minimum of the reverse curve is the turning point between the prevailing of oxidation or reduction processes. Our results are evidence that the bell-shaped dose dependences recorded for various cellular responses are characteristic also for redox changes in the photoacceptor, cytochrome c oxidase.
\end{abstract}

\section{Introduction}

$\mathbf{P}$ HOTOTHERAPY USES MONOCHROMATIC and quasimonochromatic light from lasers and light emitting diodes (LEDs) in the red to near infrared (NIR) optical region $(\sim 600-1000$ $\mathrm{nm}$ ) to treat in a nondestructive and non-thermal fashion various dermatological, soft-tissue, and neurological conditions. ${ }^{1}$ It is believed that this kind of treatment is based on the ability of monochromatic light in the red to NIR region to alter cellular metabolism as a result of its being absorbed by cytochrome c oxidase. ${ }^{2-5}$ Cytochrome c oxidase is the enzyme that catalyzes the final step in mitochondrial respira- tory chain: the transfer of electrons from cytochrome c to molecular oxygen.

Phototherapy, which is still outside the mainstream of medicine, has gained more attention in the past few years, for this method has proven useful and has great potential in various fields of medicine and bioengineering. The reduction of the infarct size following a myocardial infarction, ${ }^{6}$ decreasing methanol-induced retinal toxicity, ${ }^{4,7}$ partial rescue of visual cortical neurons from cyanide-induced apoptosis, ${ }^{8}$ promotion of the regeneration and functional recovery of tissues with poor healing potential, such as injured peripheral ${ }^{9}$ and optical ${ }^{10}$ nerves, and improvement of the integration of

\footnotetext{
${ }^{1}$ Institute of Laser and Information Technologies of Russian Academy of Sciences, ${ }^{2}$ Institute of Spectroscopy of Russian Academy of Sciences, Troitsk, Moscow Region 142190, Russian Federation, and ${ }^{3}$ SpectroOptical Sensing Inc., Madison, Wisconsin.
} 
bony $^{11}$ and titanium ${ }^{12}$ implants are but a few of the potential uses for phototherapy. Light at 670 and $830 \mathrm{~nm}$ has been shown to upgrade cytochrome c oxidase functionally inactivated with potassium cyanide in cultured primary neurons. ${ }^{3}$ Lane has recently detailed the importance of these findings. ${ }^{13}$

Earlier measurements of absorption spectra of the cell monolayer have revealed that the 710- to 790-nm spectral region is characteristic of the relatively reduced photoacceptor, while the 650- to $680-\mathrm{nm}$ one characterizes the relatively oxidized photoacceptor. ${ }^{14}$ It was found in a previous study that the ratio between the peak intensities near 760-780 nm and $660-680 \mathrm{~nm}$ could be used to characterize the redox status of cytochrome c oxidase. ${ }^{14}$ This criterion is used in the present work as well.

To further investigate the involvement of cytochrome c oxidase as the photoacceptor into modulation of cellular metabolism, we first recorded the absorption spectra of a cellular monolayer after multiple irradiations at $632.8 \mathrm{~nm}$. Second, we evaluated the changes in these absorption spectra by the criterion of ratio of peak intensities to investigate changes in the redox status of cytochrome c oxidase due to the irradiation. The wavelength $632.8 \mathrm{~nm}$ (He-Ne laser) has often been used for phototherapy. ${ }^{1}$ We intend to show that the irradiation may cause reduction or oxidation of cytochrome c oxidase, depending on the initial redox status of the enzyme.

\section{Materials and Methods \\ Cells}

The HeLa cells obtained from the Institute of Virology, Moscow, Russia, were cultivated as a monolayer in special enclosed polytetrafluoroethylene cuvettes provided with quartz windows (described in detail in reference 15) at $37^{\circ} \mathrm{C}$ in RPMI-1640 (ICN Pharmaceutical, Amsterdam, The Netherlands) with $10 \%$ fetal bovine serum (ICN Pharmaceutical) and $100 \mathrm{U} / \mathrm{mL}$ of penicillin and streptomycin. The cells were grown in $2 \mathrm{~mL}$ of the nutrient medium for $72 \mathrm{~h}$ (middle-log phase), after which a uniform monolayer covered the entire surface of the cuvette window. The HeLa culture used is characterized by anchorage-dependent growth and forms a confluent monolayer. The cells were handled and irradiated in the dark or in dim natural light.

\section{Optical measurements}

The absorption spectra of the cellular monolayer were measured using the set-up described in detail in reference 15. The spectrophotometer and optical analyzer were constructed by Dr. E. Silkis, Institute of Spectroscopy Russian Academy of Sciences, Troitsk, Russia. The analyzer was coupled to a computer that implemented signal averaging and data processing procedures. The spectral data were processed (transmission-to-absorption conversion, smoothing, baseline correction, curve fitting, and deconvolution of spectra with the Lorentzian fitting) using the Origin 7.5 (OriginLabs Corp., Northampton, MA, USA) software package. The measurements were taken immediately after the cuvette opening, or $3 \mathrm{~min}$ after the cuvette opening. The spectra were recorded in the wavelength range from $620-820 \mathrm{~nm}$.

\section{Irradiation of the cellular monolayer}

The cells were irradiated with a He-Ne laser (Spectra Physics model 125A; Mountain View, CA, USA). The laser beam parameters were as follows: wavelength $632.8 \mathrm{~nm}, \tau_{\mathrm{ir}-}$ rad. $=10 \mathrm{sec}$, dose $6.3 \times 10^{3} \mathrm{~J} / \mathrm{m}^{2}$. The position of the laser beam in the experimental set-up for absorbancy measurements was similar to that described in reference 15 .

The irradiation and measurement procedures were consecutively performed non-stop. The irradiation was performed three times in all experiments $\left(\tau_{\text {irrad. }}=10 \mathrm{sec}\right.$ and $\tau_{\text {record. }}=600 \mathrm{msec}$ ). The cuvette window was fixed during the experiment so that exactly the same area of the monolayer was irradiated and measured each time. All preparation procedures, spectral measurements, and the irradiation were performed at room temperature in the dark.

\section{Quantitative characterization of the redox status of the photoacceptor in the absorption spectra}

For the quantitative characterization, as well as for the comparison between the recorded absorption spectra, we used intensity ratios between certain fitted peaks as described in detail in reference 14 . The peak used to characterize the relative reduced photoacceptor was the one around $770 \mathrm{~nm}$ (Table 1). The peak used to characterize the relatively oxidized photoacceptor was the one around $670 \mathrm{~nm}$ (Table 1). Error bars of baseline correction and deconvolution of the spectra with the Lorentzian fitting for individual peaks are $\leq 5 \mathrm{~nm}$. The gray vertical bands in Fig. 1 mark the bands chosen. It should be mentioned that in the reference 14 the exact wavelengths chosen for the intensity ratio criterion were 760 and $665 \mathrm{~nm}$. The difference of $10 \mathrm{~nm}$ between the criterion used in that paper and that used in this one falls within the error bars of baseline correction and deconvolution of the spectra with the Lorentzian fitting. The wavelengths 770 and $670 \mathrm{~nm}$ fall well into spectral regions characteristic for the relatively reduced $(710-790 \mathrm{~nm})$ and relatively oxidized (650-680 nm) photoacceptor, as was found in reference 14.

The intensity ratio of the fitted peaks $\boldsymbol{I}_{770} / \boldsymbol{I}_{670}$ was calculated to characterize every spectrum. In the case of equal concentrations of the reduced and oxidized forms of the photoacceptor molecule, the ratio $I_{770} / I_{670}$ should be equal to unity. When the reduced forms prevail, the ratio $I_{770} / I_{670}$ is greater than unity, and it is less than unity in cases where the oxidized forms dominate.

\section{Results}

The cellular monolayer was grown in the enclosed cuvette for all our experiments. In the first series of experiments, the time between opening of the cuvette and the beginning of the experiment was $3 \mathrm{~min}$. The typical spectra in this type of experiment are marked as $A_{1}, B_{1}, C_{1}$, and $D_{1}$ in Fig. 1 . $A_{1}$ is the initial spectrum and $B_{1}, C_{1}$, and $D_{1}$ mark the spectra after the first, second, and third irradiations, respectively (Fig. 1 and Table 1). In the second series of the experiments, the experiment started immediately after the opening of the cuvette. The spectra are marked as $\mathrm{A}_{2}, \mathrm{~B}_{2}, \mathrm{C}_{2}$, and $\mathrm{D}_{2}$ in Fig. 1 and Table 1. The two experimental procedures were chosen to work well with cell cultures with relatively oxidized (experiment 1 ) and reduced forms (experiment 2 ) of cytochrome c oxidase. 
Table 1. Peak Positions in Absorption Spectra of Hela Cell Monolayers in the Red to

NIR Region as Resolved by Deconvolution of the Spectra with the Lorentzian Fitting for Two Typical Experiments $\left(\mathrm{A}_{1}-\mathrm{D}_{1}\right.$ AND $\mathrm{A}_{2}-\mathrm{D}_{2}$, in Fig. 1, Respectively)

\begin{tabular}{|c|c|c|c|c|c|c|c|c|}
\hline \multicolumn{2}{|c|}{$\begin{array}{l}\text { Initial } \\
\text { spectrum }\end{array}$} & \multicolumn{2}{|c|}{$\begin{array}{l}\text { After the first } \\
\text { irradiation }\end{array}$} & \multicolumn{2}{|c|}{$\begin{array}{l}\text { After the second } \\
\text { irradiation }\end{array}$} & \multicolumn{2}{|c|}{$\begin{array}{l}\text { After the third } \\
\text { irradiation }\end{array}$} & \multirow[b]{2}{*}{$\begin{array}{l}\text { Characterization } \\
\text { (based on ref. } 14 \text { ) }\end{array}$} \\
\hline $\begin{array}{l}A_{1} \\
\mathrm{R}^{2}=0.90\end{array}$ & $\begin{array}{c}A_{2} \\
\mathrm{R}^{2}=0.97\end{array}$ & $\begin{array}{c}B_{1} \\
\mathrm{R}^{2}=0.92\end{array}$ & $\begin{array}{c}B_{2} \\
\mathrm{R}^{2}=0.99\end{array}$ & $\begin{array}{c}C_{1} \\
\mathrm{R}^{2}=0.95\end{array}$ & $\begin{array}{c}C_{2} \\
\mathrm{R}^{2}=0.95\end{array}$ & $\begin{array}{c}D_{1} \\
\mathrm{R}^{2}=0.96\end{array}$ & $\begin{array}{c}D_{2} \\
\mathrm{R}^{2}=0.99\end{array}$ & \\
\hline 632 & 632 & 634 & 629 & 634 & 629 & 634 & 631 & Reduced photoacceptor \\
\hline 669 & 668 & 670 & 669 & 669 & 670 & 669 & 669 & $\begin{array}{l}\text { Oxidized photoacceptor } \\
\text { (gray line in Fig. 1) }\end{array}$ \\
\hline 719 & 716 & 723 & 717 & 720 & 717 & 719 & 716 & Reduced photoacceptor \\
\hline 750 & 749 & 752 & 746 & 751 & 747 & 754 & 747 & \\
\hline 770 & 771 & 776 & 772 & 771 & 773 & 770 & 773 & $\leftarrow$ (gray line in Fig. 1$)$ \\
\hline 802 & $801^{*}$ & 792 & 788 & 791 & 790 & 786 & 792 & \\
\hline 820 & 807 & 814 & 811 & 820 & 811 & 819 & 809 & Oxidized photoacceptor \\
\hline
\end{tabular}

ansufficient resolution.

$A_{1,2}$ are the spectra before and $B_{1,2}, C_{1,2}, D_{1,2}$ are the spectra after iradiation with a He-Ne laser $\left(632.8 \mathrm{~nm}, 6.3 \times 10^{3} \mathrm{~J} / \mathrm{m}^{2},-10 \mathrm{sec}\right) \mathrm{B}_{1,2}$ one, $\mathrm{C}_{1,2}$ two, or $\mathrm{D}_{1,2}$ three times.

$\mathrm{R}^{2}$, mean square deviation of fitting.

Thus Fig. 1 presents two typical initial absorption spectra $\left(\mathrm{A}_{1}\right.$ and $\left.A_{2}\right)$, and the same spectra after the first $\left(B_{1}\right.$ and $\left.B_{2}\right)$, second $\left(C_{1}\right.$ and $\left.C_{2}\right)$, and third $\left(D_{1}\right.$ and $\left.D_{2}\right)$ irradiations. Spectrum $A_{1}$ is characterized by bands resolved by the Lorentzian fitting method with peak positions at 632, 669, 719, 750, 770, 802, and $820 \mathrm{~nm}$ (Table 1). The irradiation of these cells did not cause changes in the peak positions (the changes fall into the error bars of the baseline correction and Lorentzian fitting), as evidenced by the data from spectra $B_{1}, C_{1}$, and $D_{1}$ in Table 1 .

Spectrum $\mathrm{A}_{2}$ is characterized by resolved peak positions at $632,668,716,749,771$, and $807 \mathrm{~nm}$, as well as by an insufficiently resolved weak peak at $801 \mathrm{~nm}$ (Table 1). Irradiation does not cause statistically significant changes in peak positions of this spectrum (spectra $B_{2}, C_{2}$, and $D_{2}$ in Table 1 ).

However, the irradiation causes substantial changes in peak intensities in the spectra of both series of experiments (spectra $A_{1}-D_{1}$ and $A_{2}-D_{2}$ in Fig. 1). For quantitative characterization and for comparison of the recorded spectra, we used intensity ratios between fitted peaks at 770 and $670 \mathrm{~nm}$ (Table 1) for the relatively reduced and oxidized photoacceptor, respectively (criterion $\boldsymbol{I}_{770} / \boldsymbol{I}_{670}$ ). Gray bands in Fig. 1 indicate these regions.

There is only one band resolved in the far red region in the part of the spectrum that characterizes relatively oxidized cytochrome c oxidase (peak position at $670 \mathrm{~nm}$ ). The part of the spectrum that is characteristic of the relatively reduced photoacceptor has triplet peaks at 720, 750, and $770 \mathrm{~nm}$ in this region (Table 1 ). In our calculations of criterion $I_{770} / I_{670}$ we used for characterization of a relatively reduced photoacceptor only one band of this triplet $(\lambda=770 \mathrm{~nm})$. The results when the intensities of all three peaks are used for calculating intensity ratios will be considered separately elsewhere due to the complexity of the material.

The value of the criterion $\boldsymbol{I}_{770} / \boldsymbol{I}_{670}$ is different for two initial spectra $A_{1}$ and $A_{2}$, being $0.30 \pm 0.05$ and $1.30 \pm 0.06$, respectively. Mean values $\pm S D$ were calculated from three independent measurements and are shown as error bars in Fig. 2. Recall that the value $\boldsymbol{I}_{770} / \boldsymbol{I}_{670}<1$ characterizes a relatively reduced photoacceptor, and the value $\boldsymbol{I}_{770} / \boldsymbol{I}_{670}>1$ is characteristic of a relatively oxidized photoacceptor. ${ }^{14}$ A difference in the initial values of $I_{770} / I_{670}$ in the spectra $A_{1}$ and $A_{2}$ means that a relative redox status of the photoacceptor is different in these two spectra. Spectrum $\mathrm{A}_{1}$ belongs to a relatively oxidized photoacceptor, and spectrum $\mathrm{A}_{2}$ is characteristic of a relatively reduced photoacceptor.

Irradiation at $632.8 \mathrm{~nm}$ caused different changes in spectra $A_{1}$ and $A_{2}$. In case of spectrum $A_{1}$, the criterion $\boldsymbol{I}_{770} / \boldsymbol{I}_{670}$ increased after the first and second irradiation events, which in spectra $B_{1}$ and $C_{1}$ are $0.51 \pm 0.04$ and $0.80 \pm 0.06$, respectively. The third irradiation caused a decrease in the criterion to $0.19 \pm 0.07$ (spectrum $D_{1}$ ), which is not statistically significantly different from the initial value of $\boldsymbol{I}_{770} / \boldsymbol{I}_{670}$ in spectrum $A_{1}$. So the irradiation causes first a reduction of the initially relatively oxidized photoacceptor, and then after a certain maximum $\left(\mathrm{C}_{1}\right)$, an oxidation occurs (Fig. $2 \mathrm{a}$ ).

In case of spectra $A_{2}-D_{2}$, the situation is the opposite. The initial value of the criterion $I_{770} / I_{670}$ in spectrum $A_{2}, 1.30 \pm$ 0.06 , is characteristic of a relatively reduced photoacceptor. In this case, the first and second irradiation events (spectra $B_{2}$ and $C_{2}$ ) cause a relative oxidation (the criteria $I_{770} / I_{670}$ for spectra $B_{2}$ and $C_{2}$ are $0.89 \pm 0.02$ and $0.40 \pm 0.05$, respectively). The third irradiation (spectrum $\mathrm{D}_{2}$ ) causes a slight relative reduction $\left(\boldsymbol{I}_{770} / \boldsymbol{I}_{670}=0.61 \pm 0.05\right.$; Fig. $\left.2 \mathrm{~b}\right)$. The data for points $B_{2}, C_{2}$, and $D_{2}$ are statistically significantly different from the initial value of $\boldsymbol{I}_{770} / \boldsymbol{I}_{670}$ for point $\mathrm{A}_{2}$.

\section{Discussion}

It was found in the present work that the relative redox status of the putative photoacceptor molecule, cytochrome c oxidase, is influenced by irradiation at $632.8 \mathrm{~nm}$ in dependence on its initial redox status. Depending upon the initial redox status, the irradiation can cause either relative reduction or relative oxidation.

It was suggested that the intensity ratio of bands near 760-770 and 670-680 nm may roughly characterize the re- 

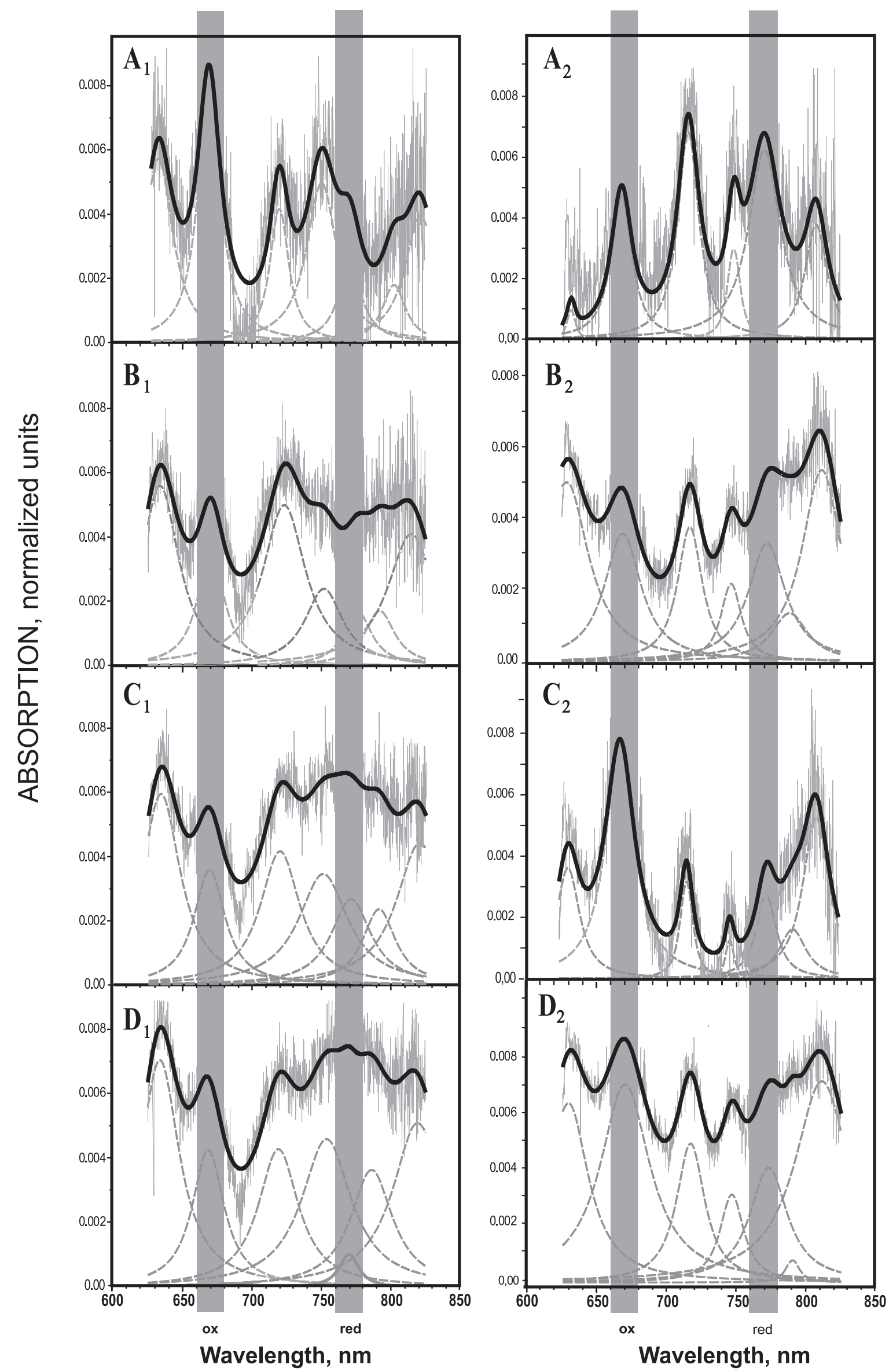

FIG. 1. 
dox status of the catalytic center in cytochrome c oxidase, [heme $\left.a_{3}-\mathrm{Cu}_{\mathrm{B}}\right] \cdot{ }^{14}$ If this is the case, then Fig. 2 represents changes in the redox status of cytochrome c oxidase's catalytic center. Fig. 2 also shows that there are some limits to the values of redox changes caused by irradiation. In our experimental conditions, these limits are the values for $\boldsymbol{I}_{770} / \mathbf{I}_{670}$ of $0.40 \pm 0.05$ and $0.80 \pm 0.06$ (the data for points $C_{1}$ and $C_{2}$ in Fig. 2). It is noteworthy that both of these values are $<1$, i.e., they belong to the photoacceptor where reduction processes are prevailing. Recall that the value $\boldsymbol{I}_{770} / \boldsymbol{I}_{670}=1.0$ means that the reduced and oxidized forms are equally present. ${ }^{14}$ This equilibrium is not reached during irradiation (Fig. 2a).

We would like to emphasize that we are working with a collection of cells, and the effects measured reflect average changes in many cytochrome c oxidase molecules in cells belonging to various subpopulations of a cell monolayer, which differ in growth rates as well as cell cycle phases (i.e., in their energy demands).

The electronic excitation of a chromophore facilitates redox processes (i.e., electrons are transferred more easily). ${ }^{16}$ Let us look for a possible chromophore that can absorb the light at the wavelength of $632.8 \mathrm{~nm}$, as used for irradiation in our experiments. It was suggested years ago on the basis of various cellular action spectra that a maximum around $620 \mathrm{~nm}$ in the action spectra may belong to $\mathrm{Cu}_{\mathrm{A}}$ in reduced form (see the review in reference 2). In various cellular action spectra, this broad band has peak positions at $624 \mathrm{~nm}$ (full width of the band at half maximum [FWHM] $30 \mathrm{~nm}$ ), $615 \mathrm{~nm}$ (FWHM $58 \mathrm{~nm}$ ), $619 \mathrm{~nm}$ (FWHM $29 \mathrm{~nm}$ ), $614 \mathrm{~nm}$ (FWHM $49 \mathrm{~nm}$ ), and $618 \mathrm{~nm}$ (FWHM $19 \mathrm{~nm}) .{ }^{17}$ The wavelength $632.8 \mathrm{~nm}$ used for irradiation in this work falls into these bands. A peak near 616 or $630 \mathrm{~nm}$ was also present in absorption spectra recorded in the cell monolayer in various oxidation conditions. ${ }^{14}$ In the present work, the peak is recorded near $630 \mathrm{~nm}$ (Table 1).

It has long been known that various cellular responses to irradiation at $632.8 \mathrm{~nm}$ occurring after retrograde mitochondrial signaling ${ }^{18,19}$ have bell-shaped dose dependences characterized by a threshold, a phase of increase, a strict maximum, and a phase of decrease. The latest summary of these data can be found in reference 18. In this work we found that the bell-shaped dose dependence is also characteristic for changes in the redox status of the putative photoacceptor, cytochrome c oxidase.

Under certain experimental conditions, dose-dependence curves like the one shown in Fig. $2 b$ are also recorded for various cellular responses. An analysis of those curves and a comparison between the two types of curves is provided in reference 5. One should note that curves like the one shown in Fig. 2b (i.e., the opposite of a bell-shaped curve) are rather rare, as compared to the bell-shaped ones.

Both types of curves mirror two processes. First, there is one reaction during which a product is created, and then it is consumed or destroyed in another reaction. This type of
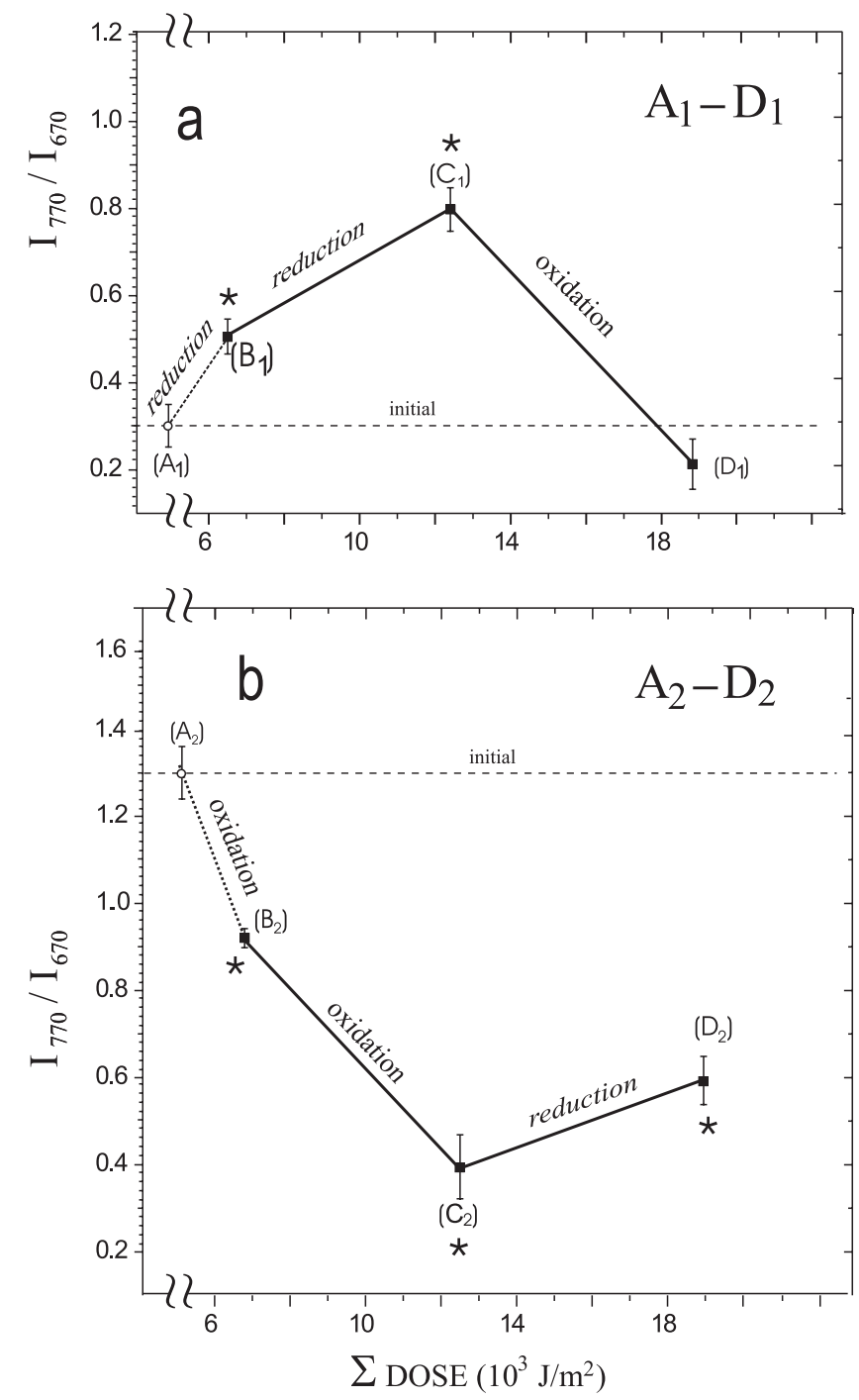

FIG. 2. Dependences of the peak intensity ratio criterion $I_{770} / I_{670}$ on the summary dose of radiation $(\lambda=632.8 \mathrm{~nm})$. (a) The points marked as $A_{1}, B_{1}, C_{1}$, and $D_{1}$ concern spectra $A_{1}, B_{1}, C_{1}$, and $D_{1}$ in Fig. 1. (b) The points marked as $A_{2}, B_{2}$, $C_{2}$, and $D_{2}$ concern spectra $A_{2}, B_{2}, C_{2}$, and $D_{2}$ in Fig. 1 . The asterisks indicate statistical significance $(p<0.05)$ of points $B_{1}, C_{1}, D_{1}$, and $B_{2}, C_{2}$, and $D_{2}$ from the initial values of points $\mathrm{A}_{1}$ and $\mathrm{A}_{2}$, respectively.

dependence is characteristic of all redox processes and corresponds to the concentrations of its oxidized and reduced components, which fall between certain borders. ${ }^{16}$ One example of a bell-shaped curve characterizing a redox process in cytochrome c oxidase is described in reference 20. Neither fully oxidized nor fully reduced cytochrome c oxidase displayed any measurable absorbance changes. The reduction levels $20 \%$ and $60 \%$ were the borders of when regulation

FIG. 1. Absorption spectra of HeLa cell monolayers: $A_{1}$ and $A_{2}$ are the initial spectra differing by redox status of the photoacceptor. The spectra of the same monolayer immediately after irradiation $\left(632.8 \mathrm{~nm}, 6.3 \times 10^{3} \mathrm{~J} / \mathrm{m}^{2}, 10 \mathrm{sec}\right): \mathrm{B}_{1}$ and $\mathrm{B}_{2}$ are the first irradiation, $C_{1}$ and $C_{2}$ are the second irradiation, and $D_{1}$ and $D_{2}$ are the third irradiation. The original spectra after baseline correction, curve fitting (-), and deconvolution of the spectra with the Lorentzian fitting (----) are shown. ox, oxidation; red, reduction. 
occurred. The flash-induced electrogenic events and absorbance changes in cytochrome c oxidase were intrinsic to the cytochrome c oxidase molecule, and the authors believed that they were related to intermediate steps in the reduction of oxygen. In addition, the reaction producing the absorbance changes was sensitive to ligands that were bound in the catalytic center $\left[\mathrm{a}_{3}-\mathrm{Cu}_{\mathrm{B}}\right]$ of cytochrome c oxidase. ${ }^{20}$

Various wavelengths can be used to cause photobiological effects in cytochrome c oxidase. These wavelengths can be in blue-to-green ${ }^{20-23}$ or red-to-near IR ${ }^{24-26}$ regions. Various wavelengths in the red-to-near IR region (most frequently 632.8, 670-680, 780, and 820-830 nm) are used in laser phototherapy, as well as in cellular studies of the mechanisms of action of this modality (see reference 5 for a review). One explanation to this puzzle could be that there exists a universal mechanism in play after chromophores of cytochrome c oxidase are electronically excited. Recall that cytochrome c oxidase has absorption bands not only in red and near IR, but also in blue and green regions. One possibility is that the radical NO bound in the catalytic center of cytochrome c oxidase is dissociated when the enzyme is reduced by irradiation, thus reversing the signaling consequences of excessive NO binding. ${ }^{27,28,13}$ This mechanism could explain first the possibility of using different wavelengths for laser phototherapy, and second, at least to some extent, a universal mechanism behind the actions of laser phototherapy.

\section{Conclusion}

The experimental results of this work demonstrate that irradiation at $632.8 \mathrm{~nm}$ causes either a (transient) relative reduction of the photoacceptor, putatively cytochrome c oxidase, or its (transient) relative oxidation, depending on the initial redox status of the photoacceptor. The maximum in the bellshaped dose-dependence curve or the minimum of the reverse bell-shaped curve is the turning point between the prevalence of an oxidation or reduction process. Our results are evidence that bell-shaped dose dependences recorded for various cellular responses occurring after mitochondrial signaling are also characteristic for redox changes in the photoacceptor cytochrome c oxidase located in the mitochondria.

\section{Acknowledgment}

This work was partly supported by grant 07-02-00205-a from the Russian Foundation of Basic Research.

\section{Disclosure Statement}

No conflicting financial interests exist.

\section{References}

1. Tunér, J., and Hode, L. (2002). Laser Therapy. Grängesberg, Sweden: Prima Books AB.

2. Karu, T. (1999). Primary and secondary mechanisms of action of visible-to-near IR radiation of cells. J. Photochem. Photobiol. B. Biol. 49, 1-17.

3. Wong-Riley, M.T.T., Liang, H.L., Eells, J.T., et al. (2005). Photobiomodulation directly benefits primary neurons functionally inactivated by toxins. J. Biol. Chem. 280, 4761-4771.

4. Eells, J.T., Henry, M.M., Summerfelt, P., et al. (2003). Ther- apeutic photobiomodulation for methanol-induced retinal toxicity. Proc. Natl. Acad. Sci. USA. 100, 3439-3444.

5. Karu, T. (2007). Ten Lectures on Basic Science of Laser Phototherapy. Grängesberg, Sweden: Prima Books AB.

6. Oron, U., Yaakobi, T., Oron, A., et al. (2001). Low energy laser irradiation reduces formation of scar tissue following myocardial infarction of dogs. Circulation. 103, 296-301.

7. Eells, J., Wong-Riley, M.T., Verhover, J., et al. (2004). Mitochondrial signal introduction in accelerated wound and retinal healing by near-infrared light therapy. Mitochondrion. 4, 559-567.

8. Liang, H.L., Whelan, H.T., Eells, J.T., et al. (2006). Photobiomodulation partially rescues visual cortical neurons from cyanide-induced apoptosis. Neuroscience. 139, 639-649.

9. Anders, J., Geuna, S., and Rochkind, S. (2004). Phototherapy promotes regeneration and functional recovery of injured peripheral nerve. Neurol. Res. 26, 234-240.

10. Assia, E., Rosner, M., Belkin, M., Solomon, A., and Schwartz, M. (1989). Temporal parameters of low energy laser irradiation for optimal delay of post-traumatic degeneration of rat optic nerve. Brain Res. 476, 112-205.

11. Dörtbudak, O., Haas, R., and Mailath-Pokorny, G. (2002). Effect of low-power laser irradiation on bony implant sites. Oral. Implants Res. 13, 288-292.

12. Kharda, M., Lyngstadaas, S.P., Haanes, H.R., and Mustafa, K. (2005). Effect of laser therapy on attachment, proliferation and differentiation of human osteoblast-like cells cultured on titanium implant material. Biomaterials. 26, 3503-3509.

13. Lane, N. (2006). Power games. Nature. 443, 901-903.

14. Karu, T.I., Pyatibrat, L.V., Kolyakov, S.F., and Afanasyeva, N.I. (2005). Absorption measurements of a cell monolayer relevant to phototherapy: reduction of cytochrome c oxidase under near IR radiation. J. Photochem. Photobiol. B. Biol. 81, 98-106.

15. Karu, T.I., Afanasyeva, N.I., Kolyakov, S.F., Pyatibrat, L.V., and Welser, L. (2001). Changes in absorbance of monolayer of living cells induced by laser radiation at 633,670, and 820 nm. IEEE J. Select. Topics Quantum Electr. 7, 982-988.

16. Marcus, R.A., and Sutin, N. (1984). Electron transfers in chemistry and biology. Biochim. Biophys. Acta. 811, 265-322.

17. Karu, T.I., and Kolyakov, S.F. (2005). Exact action spectra for cellular responses relevant to phototherapy. Photomed. Laser Surg. 23, 355-361.

18. Karu, T.I. (2008). Mitochondrial signaling in mammalian cells activated by red and near IR radiation. Photochem Photobiol 84, 1091-1099.

19. Schroeder, P., Pohl, C., Calles, C., Marks, C., Wild, S., and Krutmann, J. (2007). Cellular response to infrared radiation involves retrograde mitochondrial signaling. Free Rad. Biol. Med. 43, 128-135.

20. Hallén, S., and Brzezinski, P. (1994). Light-induced structural changes in cytochrome c oxidase: implication for the mechanism of electron and proton gating. Biochim. Biophys. Acta 1184, 207-218.

21. Zaslavsky, D., Kaulen, A.D., Smirnova, I.A., Vygodina, A.A., and Konstantinov, A.A. (1993). Flash-induced membrane potential generation by cytochrome c oxidase. FEBS Lett. 336, 389-393.

22. Adar, F., and Yonetani, T. (1978). Resonance Raman spectra of cytochrome c oxidase evidence for photoreduction by 
laser photons in resonance with the Soret band. Biochim. Biophys. Acta 502, 80-86.

23. Salet, C., Moreno, G., and Vinzens, F. (1979). A study of beating frequency of a single myocardial cell. III. Laser microirradiation of mitochondria in the presence of $\mathrm{KCN}$ and ATP. Exp. Cell Res. 120, 25-29.

24. Arvanitaki, A., and Chalazonitis, N. (1947). Reactiones bioélectriques á la photoactivation des cytochromes. Arch. Sci. Physiol. 1, 385-405.

25. Kato, M., Shinzawa, K., and Yoshikawa, S. (1981). Cytochrome oxidase is a possible photoacceptor in mitochondria. Photochem. Photobiophys. 2, 263-269.

26. Pastore, D., Greco, M., and Passarella, S. (2000). Specific HeNe laser sensitivity of the purified cytochrome c oxidase. Int. J. Rad. Biol. 76, 863-870.
27. Karu, T., Pyatibrat, L., and Afanasyeva, N. (2004). A novel mitochondrial signaling pathway activated by visible-tonear infrared radiation. Photochem. Photobiol. 80, 366-372.

28. Karu, T.I., Pyatibrat, L.V., and Afanasyeva, N.I. (2005). Cellular effects of low power laser therapy can be mediated by nitric oxide. Lasers Surg. Med. 36, 307-314.

Address reprint requests to: Prof. T.I. Karu, Dr. Sci., Ph.D. Institute of Laser and Information Technologies of Russian Academy of Sciences Pionerskaya Str. 2 Troitsk 142190, Moscow Region, Russian Federation E-mail: tkaru@isan.troitsk.ru 